\title{
On stability of forcing relations for multidimensional perturbations of interval maps
}

\author{
by \\ Ming-Chia Li (Hsinchu) and Piotr Zgliczyński (Kraków) \\ Dedicated to the 60th birthday of Michat Misiurewicz
}

\begin{abstract}
We show that all periods of periodic points forced by a pattern for interval maps are preserved for high-dimensional maps if the multidimensional perturbation is small. We also show that if an interval map has a fixed point associated with a homocliniclike orbit then any small multidimensional perturbation has periodic points of all periods.
\end{abstract}

1. Introduction. For continuous interval maps, the Šarkovskiı̌ theorem $[7,8]$ is the complete answer to the following problem: Given a periodic orbit of a specified period, find the other periods of periodic orbits that must exist. One can classify patterns of orbits depending on the arithmetic ordering of the points on the real line (Definition 2.1), and consider the so-called forcing problem: Given a period- $n$ orbit of a specified pattern, find, for any positive integer $m$, the patterns of period- $m$ orbits that must exist. The forcing problem is far from being completely solved yet. So far, there are some partial results giving forcing relations such as: A periodic cycle with a certain pattern forces the existence of periodic cycles with other patterns. In fact, considering patterns characterized by one parameter, a chain of forcing relations established in $[2,3,5,9]$ provides a refinement of the Šarkovskiu theorem (see also [6, Theorem 2]). A further generalization to patterns characterized by two parameters was given in [6, Theorem 3]. For more discussions on the forcing problem, refer to [1] and [3].

In this paper, we study the stability of forcing relations for one-dimensional maps with respect to multidimensional perturbations. More precisely, let $F_{\lambda}$ be a one-parameter family of continuous maps on $\mathbb{R} \times \mathbb{R}^{n}$ such that $F_{\lambda}(z)$ is continuous as a function jointly of $\lambda$ and $z$, where $\lambda \in \mathbb{R}$ is a parameter,

2010 Mathematics Subject Classification: 37E15, 37C25, 37B30.

Key words and phrases: forcing relations, multidimensional perturbation, patterns, covering relations. 
and at $\lambda=0$, the map $F_{0}$ is of one of the following forms:

(i) $F_{0}(x, y)=(f(x), g(x)) \in \mathbb{R} \times \mathbb{R}^{n}$, where $f: \mathbb{R} \rightarrow \mathbb{R}$ and $g: \mathbb{R} \rightarrow \mathbb{R}^{n}$ are continuous functions;

(ii) $F_{0}(x, y)=(f(x), g(x, y)) \in \mathbb{R} \times \mathbb{R}^{n}$, where $f: \mathbb{R} \rightarrow \mathbb{R}$ is continuous and $g: \mathbb{R} \times \mathbb{R}^{n} \rightarrow \mathbb{R}^{n}$ is continuous and $g(\mathbb{R} \times S) \subset \operatorname{int}(S)$ for some compact set $S \subset \mathbb{R}^{n}$ homeomorphic to the closed unit ball in $\mathbb{R}^{n}$.

A natural question is which periods (or patterns) of periodic points the map $F_{\lambda}$ can have when $f$ has a certain period (or pattern) of a periodic point and $|\lambda|$ is small.

For the case when $g$ is the zero function, it was shown in [10] that if $f$ has a periodic point of period $k$, then for all sufficiently small $|\lambda|$, the map $F_{\lambda}$ has a periodic point of period less than $k$ in the Šarkovskiı̌ ordering.

In the present paper, we remove the constraint $g=0$ and consider perturbations of a larger class of maps, including the Hénon map $F_{b}(x, y)=$ $\left(a-x^{2}+b y, x\right)$. We show which periods of periodic points for $F_{\lambda}$ are forced by a given pattern for $f$. More precisely, first we show that if $f$ has a periodic point of any given pattern $A$, then for all sufficiently small $|\lambda|$, the map $F_{\lambda}$ has periodic points of periods of patterns forced by $A$ (Theorem 2.2). Second, we show that if there are two points $\alpha$ and $\beta$ such that either $f(\alpha) \leq \alpha<\beta<f(\beta)<f^{2}(\beta)$ and $f^{3}(\beta) \leq \alpha$, or the same with all inequalities reversed, then for all sufficiently small $|\lambda|$, the map $F_{\lambda}$ has periodic points of all periods (Theorem 2.3). In particular, one can consider the case when $f$ has a fixed point associated with a homoclinic-like point (Example 6.1). Theorem 2.2 extends the result in [10] because of the Šarkovskii theorem. In the sense of multidimensional perturbations, Theorem 2.3 extends the results in $[2,5,6]$ which concern a fixed point with homoclinic orbits of certain patterns. The covering relations approach is the main tool for the proof of our results.

The paper is organized as follows. In the next section, we state the main results (Theorems 2.2 and 2.3) precisely along with the definition of patterns. In Section 3, we state the Šarkovskiı theorem and a stability result for multidimensional perturbations. In Section 4, we recall the notion of covering relation and some related results. In Sections 5 and 6, we give the proof of Theorems 2.2 and 2.3 along with respective examples.

2. Definitions and statement of theorems. First of all, we set up some notations. For convenience, we will write $V=\mathbb{R} \times \mathbb{R}^{n}$ and represent elements $v \in V$ as pairs $v=(x, w)$, where $x \in \mathbb{R}$ and $w \in \mathbb{R}^{n}$. For a map $F: \mathbb{R} \times V \rightarrow V$, we will use the notation $F_{\lambda}$ for the partial map with fixed $\lambda \in \mathbb{R}$, so $F_{\lambda}(v):=F(\lambda, v)$ for $v \in V$. For a subset $S$ of a metric space, let $\bar{S}$, $\operatorname{int}(S)$ and $\partial S$ denote the closure, interior and boundary of $S$, respectively. 
Let us fix any norm $\|\cdot\|$ on $\mathbb{R}^{n}$. For $r>0$, we denote by $B_{n}(r)$ the open ball of radius $r$ centered at the origin in $\mathbb{R}^{n}$, i.e. $B_{n}(r)=\left\{w \in \mathbb{R}^{n}:\|w\|<r\right\}$, and by $\mathcal{C}(r)$ the family of cylinders of the form $[a, b] \times \overline{B_{n}(r)}$. When $n=0$ the above notations for $V$ and $C(r)$ do not make sense and we let $V=\mathbb{R}$ and $C(r)$ be the set of all closed proper intervals, for any $r$.

Next, we define forcing relations and some basic terminology (refer to [1]).

Definition 2.1. We say that $(P, \varphi)$ is a cycle if $P \subset \mathbb{R}$ is a finite nonempty set and $\varphi$ is a cyclic permutation of $P$. The number of elements of $P$ will be denoted by $|P|$ and will be called the period of $(P, \varphi)$. We also denote by $\langle P\rangle$ the smallest closed interval in $\mathbb{R}$ containing $P$. An interval is said to be proper if it contains more than one point. If $f$ is a continuous map on $\mathbb{R}$ and $(P, \varphi)$ is a cycle, we say that $f$ has a cycle $P$ is $\varphi=\left.f\right|_{P}$, the restriction of $f$ to $P$.

Let $\mathfrak{P}$ be the set of all cycles in $\mathbb{R}$. We define two equivalence relations in $\mathfrak{P}$ as follows. Let $(P, \varphi),(Q, \psi) \in \mathfrak{P}$. We say that $(P, \varphi) \underset{\text { pat }}{\sim}(Q, \psi)$ if and only if there exists a homeomorphism $h:\langle P\rangle \rightarrow\langle Q\rangle$ such that $h(P)=Q$ and $\left.\psi \circ h\right|_{P}=\left.h \circ \varphi\right|_{Q}$. If additionally $h$ can be chosen in such a way that it preserves orientation, then we write $(P, \varphi) \underset{\text { opat }}{\sim}(Q, \psi)$. Equivalence classes of the relation $\underset{\text { pat }}{\sim}($ resp. $\underset{\text { opat }}{\sim})$ in $\mathfrak{P}$ will be called patterns (resp. oriented patterns).

If $A$ is a pattern (resp. oriented pattern) and $(P, \varphi) \in A$ we say that the cycle $P$ has pattern $A$ (resp. oriented pattern $A$ ), and the period of $A$ is defined to be $|P|$ and will be denoted by $|A|$. An oriented pattern $B$ is called a component of a pattern $A$ if there exists a cycle which has both oriented pattern $B$ and pattern $A$.

The forcing relations between patterns and oriented patterns are defined as follows. Let $A$ and $B$ be two patterns (resp. oriented patterns). We say that $A$ forces $B$ and write $A \underset{\text { pat }}{\Longrightarrow} B$ (resp. $A \underset{\text { opat }}{\Longrightarrow} B$ ) if every continuous map on $\mathbb{R}$ which has a cycle with pattern $A$ (resp. oriented pattern $A$ ) has a cycle with pattern $B$ (resp. oriented pattern $B)$.

For a pattern $A$, let $\operatorname{Per}(A)=\{|B|: B \neq A$ is a pattern such that $A \underset{\mathrm{pat}}{\Longrightarrow} B\}$.

Now, we state the main results. The first one is that all periods of periodic points forced by a pattern for interval maps are preserved for highdimensional maps if the multidimensional perturbation is small.

THEOREM 2.2. Let $F: \mathbb{R} \times V \rightarrow V$ be a continuous function such that for all $(x, y) \in V$, either (i) $F_{0}(x, y)=(f(x), g(x))$, where $f$ is continuous on $\mathbb{R}$ and $g$ is a continuous function from $\mathbb{R}$ to $\mathbb{R}^{n}$, or (ii) $F_{0}(x, y)=$ $(f(x), g(x, y))$, where $f$ is continuous on $\mathbb{R}$ and $g$ is continuous on $\mathbb{R} \times S$ and $g(\mathbb{R} \times S) \subset \operatorname{int}(S)$, for some compact set $S \subset \mathbb{R}^{n}$ homeomorphic to the closed 
unit ball in $\mathbb{R}^{n}$. If $f$ exhibits a pattern $A$, then there exists a positive constant $\lambda_{0}$ such that if $|\lambda|<\lambda_{0}$ then $F_{\lambda}$ has periodic points of all periods in $\operatorname{Per}(A)$.

The second result shows that any small multidimensional perturbation of an interval map with $f(\alpha) \leq \alpha<\beta<f(\beta)<f^{2}(\beta)$ and $f^{3}(\beta) \leq \alpha$ has periodic points of all periods.

TheOREM 2.3. Let $F: \mathbb{R} \times V \rightarrow V$ be a continuous function such that for all $(x, y) \in V$, either (i) $F_{0}(x, y)=(f(x), g(x))$, where $f$ is continuous on $\mathbb{R}$ and $g$ is a continuous function from $\mathbb{R}$ to $\mathbb{R}^{n}$, or (ii) $F_{0}(x, y)=$ $(f(x), g(x, y))$, where $f$ is continuous on $\mathbb{R}$ and $g$ is continuous on $\mathbb{R} \times S$ and $g(\mathbb{R} \times S) \subset \operatorname{int}(S)$, for some compact set $S \subset \mathbb{R}^{n}$ homeomorphic to the closed unit ball in $\mathbb{R}^{n}$. If there are two points $\alpha$ and $\beta$ in $\mathbb{R}$ such that either $f(\alpha) \leq \alpha<\beta<f(\beta)<f^{2}(\beta)$ and $f^{3}(\beta) \leq \alpha$, or the same with all inequalities reversed, then there exists a positive constant $\lambda_{0}$ such that if $|\lambda|<\lambda_{0}$ then $F_{\lambda}$ has periodic points of all periods.

3. Šarkovskiı̌ order. The Šarkovskiı̆ theorem $[7,8]$ yields forcing relations on periods for interval maps.

THeOREM 3.1. Let $f: I \rightarrow \mathbb{R}$ be a continuous map. If $n \triangleright k$ and $f$ has a periodic point of period $n$ then $f$ also has a periodic point of period $k$, where $\triangleright$, called the Sarkovskiı ordering of positive integers, is defined as follows:

$$
\begin{gathered}
3 \triangleright 5 \triangleright 7 \triangleright \cdots \triangleright 2 \cdot 3 \triangleright 2 \cdot 5 \triangleright 2 \cdot 7 \triangleright \cdots \triangleright 2^{2} \cdot 3 \triangleright 2^{2} \cdot 5 \triangleright 2^{2} \cdot 7 \triangleright \\
\quad \cdots \triangleright 2^{i} \cdot 3 \triangleright 2^{i} \cdot 5 \triangleright 2^{i} \cdot 7 \triangleright \cdots \triangleright 2^{j} \triangleright 2^{j-1} \triangleright \cdots \triangleright 2^{2} \triangleright 2 \triangleright 1 .
\end{gathered}
$$

In [10] it was shown that the forcing relation on periods is stable with respect to multidimensional perturbations of 1-dim maps. The precise statement is

Theorem 3.2. Let $f: \mathbb{R} \rightarrow \mathbb{R}$ be continuous and $F:[0,1] \times V \rightarrow V$ be continuous with $F_{0}(x, w)=(f(x), 0)$. If $f$ has a periodic point of period $k$, then for any $r>0$, there exists $\lambda_{0}>0$ such that for all $0 \leq \lambda \leq \lambda_{0}$ and $m \neq k$ with $k \triangleright m$, the map $F_{\lambda}$ has a periodic point of period $m$ in the set $\mathbb{R} \times B_{n}(r)$.

The proof of the above theorem uses the notion of covering relation in multidimensional situation and the continuation of 1-dimensional orbits with nonzero fixed point index for multidimensional perturbations of 1dimensional maps. This technique is recalled in Section 4 .

4. Covering relations and continuation. In this section, we define the notion of covering relation and introduce some related results which will be used in the proof of the main results. In our presentation we follow [12], where the results from [10] have been restated in a more readable form. 
For a cylinder $N=[a, b] \times \overline{B_{n}(r)} \in \mathcal{C}(r)$, we set

$$
\begin{aligned}
L(N) & =\{a\} \times \overline{B_{n}(r)}, \text { the left lid of } N, \\
R(N) & =\{b\} \times \overline{B_{n}(r)}, \text { the right lid of } N, \\
H(N) & =[a, b] \times \partial B_{n}(r), \text { the horizontal boundary of } N, \\
S_{L}(N) & =(-\infty, a) \times B_{n}(r), \text { the left side of } N, \\
S_{R}(N) & =(b, \infty) \times B_{n}(r), \text { the right side of } N .
\end{aligned}
$$

Definition 4.1. Let $N_{0}, N_{1} \in \mathcal{C}(r)$ and $G: V \rightarrow V$ be continuous. We say that $N_{0} G$-covers $N_{1}$ (horizontally) and write $N_{0} \stackrel{G}{\Rightarrow} N_{1}$ if

$$
G\left(N_{0}\right) \subset(-\infty, \infty) \times B_{n}(r)
$$

and one of the following two conditions holds:

$$
\begin{aligned}
& G\left(L\left(N_{0}\right)\right) \subset S_{L}\left(N_{1}\right) \text { and } G\left(R\left(N_{0}\right)\right) \subset S_{R}\left(N_{1}\right) \text {, } \\
& G\left(L\left(N_{0}\right)\right) \subset S_{R}\left(N_{1}\right) \text { and } G\left(R\left(N_{0}\right)\right) \subset S_{L}\left(N_{1}\right) \text {. }
\end{aligned}
$$

Condition (4.1) means that the image of $N_{0}$ under $G$ is contained in the "horizontal" strip defined by $N_{1}$. Conditions (4.2) and (4.3) mean that the left and right lids of $N_{0}$ are mapped to different sides of $N_{1}$.

We would like to adapt the above notion of horizontal covering to onedimensional maps.

Definition 4.2. Let $N_{0}, N_{1}$ be closed intervals and $G: N_{0} \rightarrow \mathbb{R}$. We say that $N_{0}$ G-covers $N_{1}$ (horizontally) and write $N_{0} \stackrel{G}{\Rightarrow} N_{1}$ if one of the following two conditions holds:

$$
\begin{array}{lll}
G\left(L\left(N_{0}\right)\right) \subset S_{L}\left(N_{1}\right) & \text { and } & G\left(R\left(N_{0}\right)\right) \subset S_{R}\left(N_{1}\right) \\
G\left(L\left(N_{0}\right)\right) \subset S_{R}\left(N_{1}\right) & \text { and } & G\left(R\left(N_{0}\right)\right) \subset S_{L}\left(N_{1}\right),
\end{array}
$$

where $L([a, b])=\{a\}, R([a, b])=\{b\}, S_{L}([a, b])=(-\infty, a), S_{R}([a, b])=$ $(b, \infty)$.

The following result is the main tool based on covering relations, which will be used to obtain periodic orbits.

Theorem 4.3 ([10, Theorem 4]). Let $N_{i} \in \mathcal{C}(r)$ and $G_{i}: V \rightarrow V$ for $0 \leq i \leq l$. Suppose that

$$
N_{0} \stackrel{G_{0}}{\Longrightarrow} N_{1} \stackrel{G_{1}}{\Longrightarrow} N_{2} \stackrel{G_{2}}{\Longrightarrow} \cdots \stackrel{G_{l-1}}{\Longrightarrow} N_{l} \stackrel{G_{l}}{\Longrightarrow} N_{0} .
$$

Then there exists $x \in \operatorname{int}\left(N_{0}\right)$ such that

$$
G_{i} \circ G_{i-1} \circ \cdots \circ G_{0}(x) \in \operatorname{int}\left(N_{i+1}\right) \quad \text { for } 0 \leq i \leq l-1
$$

and

$$
G_{l} \circ G_{l-1} \circ \cdots \circ G_{0}(x)=x .
$$




\subsection{Nested sequences of topological horseshoes}

Definition 4.4. If $n>0$, then we assume that $G:(-\infty, \infty) \times \overline{B_{n}(r)} \rightarrow$ $(-\infty, \infty) \times B_{n}(r)$ is continuous. If $n=0$ we assume $G:(-\infty, \infty) \rightarrow$ $(-\infty, \infty)$ is continuous.

Let $l \in \mathbb{N}$ and $k \in \mathbb{N} \cup\{\infty\}$ be such that $0<l<k$. Let $\left\{N_{i}^{s} \in \mathcal{C}(r)\right.$ : $i \in\{0,1\}$ and $l \leq s \leq k, s \in \mathbb{N}\}$ be a family of cylinders such that $\operatorname{int}\left(N_{0}^{l}\right) \cap$ $\operatorname{int}\left(N_{1}^{l}\right)=\emptyset$ and $N_{i}^{s} \supset N_{i}^{s+1}$ for $i \in\{0,1\}$ and $l \leq s \leq k$. We say that $G$ has an $(l, k)$-nested sequence of topological horseshoes if for all $i \in\{0,1\}$ and $l \leq s \leq k, N_{i}^{s}$ horizontally $G^{s}$-covers both the cylinders $N_{0}^{l}$ and $N_{1}^{l}$.

Theorem 4.5 ([10]). Let $G:(-\infty, \infty) \times \overline{B_{n}(r)} \rightarrow(-\infty, \infty) \times B_{n}(r)$ be a continuous map and $p \geq 2$ be an integer. If $G$ has a $(p, 2 p-1)$-nested sequence of topological horseshoes, then there exists an integer $m(p)$ such that $G$ has periodic points of all periods greater than $m(p)$.

For example $m(2)=6$ and $m(5)=30$. The proof of this theorem is based on Theorem 4.3 and several arithmetical lemmas; see [10].

4.2. 1-dim coverings. Let us fix a 1-dimensional continuous map $f$. We define a one-dimensional $f$-covering relation between segments.

Definition 4.6. Let $I=[a, b]$ and $J$ be two intervals. We denote $I \stackrel{f,+1}{\longrightarrow} J$ if $f(a)<f(b)$ and $J \subset[f(a), f(b)]$, and denote $I \stackrel{f,-1}{\longrightarrow} J$ if $f(b)<f(a)$ and $J \subset[f(b), f(a)]$. We say that $I f$-covers $J$ and write $I \stackrel{f}{\rightarrow} J$ if either $I \stackrel{f,+1}{\longrightarrow} J$ or $I \stackrel{f,-1}{\longrightarrow} J$.

The above definition of covering relation for interval maps differs from the standard one presented in [4] in two ways: here we require that the endpoints of $I$ are mapped to different sides of $J$ and we introduce a sign. Both those features will be exploited in Section 4.3 devoted to the continuation of individual periodic orbits.

Let us remark that the relation of horizontal covering is stronger than the 1-dim covering: we have

$$
\text { if } I \stackrel{f}{\Rightarrow} J \text { then } I \stackrel{f}{\rightarrow} J,
$$

but there is no implication in the reverse direction (as an example, consider the identity map). We need some condition which will guarantee the reverse implication. The following trivial lemma presents such a condition.

LEMma 4.7. Let $I$ and $J$ be closed intervals with $I \stackrel{f}{\rightarrow} J$. Then $I \stackrel{f}{\Rightarrow} K$ for any closed interval $K$ with $K \subset \operatorname{int}(J)$.

The following lemma is contained implicitly in the proof of Theorem 2.8.1 in [1]; in fact, it is an immediate consequence of Lemmas 2.6.9, 2.6.10, and 
2.6.12 therein. It demonstrates that a forcing relation for interval maps implies existence of a closed loop of covering relations (in the sense of Definition 4.6).

Lemma 4.8. Let $f: \mathbb{R} \rightarrow \mathbb{R}$ be a continuous map having a cycle with oriented pattern $A$ and assume that $B \neq A$ is an oriented pattern and $A \underset{\text { opat }}{\Longrightarrow} B$. Then the map $f$ has a cycle $Q$ with oriented pattern $B$. Moreover, there exists $z \in \tilde{Q}$ and closed proper intervals $J_{0}, J_{1}, \ldots, J_{|Q|}$ such that the following loop of 1-dim covering relations holds:

$$
J_{0} \stackrel{f}{\rightarrow} J_{1} \stackrel{f}{\rightarrow} \cdots \stackrel{f}{\rightarrow} J_{|Q|-1} \stackrel{f}{\rightarrow} J_{|Q|}
$$

and

$$
\begin{aligned}
J_{0} \subset J_{|Q|}, & \\
J_{i} \cap J_{j}=\emptyset & \text { for all } 0 \leq i, j \leq|Q|-1 \text { with } i \neq j, \\
f^{i}(z) \in J_{i} & \text { for all } 0 \leq i \leq|Q|, \\
f^{|Q|}(t) \neq t & \text { for all } t \in J_{0} \cap \partial\left(J_{|Q|}\right) .
\end{aligned}
$$

We also need the following lemma for the proof of our main results:

Lemma 4.9. Let $f: \mathbb{R} \rightarrow \mathbb{R}$ be a continuous map such that $f$ has no periodic point of period different from a power of 2 and $f$ exhibits a pattern $A$ of period $2^{k}$ for some integer $k \geq 0$. If $B$ is a pattern forced by $A$, then the period of $B$ is $2^{i}$ for some integer $0 \leq i \leq k$.

Proof. Let $f$ exhibit the oriented pattern $\tilde{A}$ for one of the components $\tilde{A}$ of $A$. By Corollary 2.7.1 of [1], $\tilde{A} \underset{\text { opat }}{\Longrightarrow} \tilde{B}$ for one of the components $\tilde{B}$ of $B$. Since $f$ has no periodic point of period different from a power of 2 , by Corollary 2.12.5 of [1], $\tilde{A}$ is primary. By Theorem 2.11 .1 of [1], $\tilde{A}$ is simple. Since $|\tilde{A}|=2^{k}$, by Lemma 2.11 .5 of $[1],|\tilde{B}|=2^{i}$ for some $i \leq k$.

4.3. Continuation. Let $f, g, F_{\lambda}$ be as in Theorems 2.2 or 3.2 with (i) $F_{0}(x, y)=(f(x), g(x))$ or (ii) $F_{0}(x, y)=(f(x), g(x, y))$.

Lemma 4.10. Let $p$ be a positive integer. Suppose that there exist intervals $I_{0}, I_{1}, \ldots, I_{p-1}$ such that the following covering relations for $f$ hold:

$$
I_{0} \stackrel{f, \epsilon_{1}}{\longrightarrow} I_{1} \stackrel{f, \epsilon_{2}}{\longrightarrow} \cdots \stackrel{f, \epsilon_{p}}{\longrightarrow} I_{0} \quad \text { with } \epsilon_{1} \ldots \epsilon_{p}=-1,
$$

where $\epsilon_{i} \in\{-1,1\}$ for all $1 \leq i \leq p$. Let $I=\bigcup_{i=0}^{p-1} I_{i}$ and $r$ be a positive number greater than the maximum of $\|g\|$ on $I$ for case (i) and on $I \times S$ for case (ii). Then there exists $\lambda_{0}>0$ such that for $|\lambda|<\lambda_{0}$, the map $F_{\lambda}$ has a periodic point $z_{\lambda}$ such that

$$
F_{\lambda}^{i}\left(z_{\lambda}\right) \in \operatorname{int}\left(I_{i}\right) \times B_{n}(r) \quad \text { for all } 0 \leq i \leq p-1, \quad F_{\lambda}^{p}\left(z_{\lambda}\right)=z_{\lambda} .
$$


The above lemma is adapted from [10, Theorem 14] in which $g$ is assumed to be the zero function and $r$ is any positive number; the proof given there also applies here if we take $r$ as above.

In the one-dimensional situation this lemma expresses the method of obtaining periodic points in the standard proof of the Šarkovskil theorem (see for example [7], [9]). In this case the assumption concerning the product of $\epsilon_{i}$ 's in (4.6) can be dropped.

5. The proof of Theorem 2.2 and an example. First, let us observe that Theorem 3.2 is also valid for $F_{\lambda}$ satisfying the assumptions of Theorem 2.2. Namely, we have the following

TheOREM 5.1. Let $F: \mathbb{R} \times V \rightarrow V$ be a continuous function such that for all $(x, y) \in V$, either (i) $F_{0}(x, y)=(f(x), g(x))$, where $f$ is continuous on $\mathbb{R}$ and $g$ is a continuous function from $\mathbb{R}$ to $\mathbb{R}^{n}$, or (ii) $F_{0}(x, y)=$ $(f(x), g(x, y))$, where $f$ is continuous on $\mathbb{R}$ and $g$ is continuous on $\mathbb{R} \times S$ and $g(\mathbb{R} \times S) \subset \operatorname{int}(S)$, for some compact set $S \subset \mathbb{R}^{n}$ homeomorphic to the closed unit ball in $\mathbb{R}^{n}$. If $f$ has a periodic orbit of period $k$ in a compact interval $I$, than for any number $r$ greater than the maximum of $\|g\|$ on $I$ for case (i) and on $I \times S$ for case (ii), there exists $\lambda_{0}>0$ such that for all $|\lambda| \leq \lambda_{0}$ and $m \neq k$ with $k \triangleright m$, the map $F_{\lambda}$ has a periodic point of period $m$ in the set $\mathbb{R} \times B_{n}(r)$.

With the choice of $r$ as above, the proof of Theorem 3.2 from [10] is also valid in the above situation.

We are in a position to prove the first main result.

Proof of Theorem 2.2. Let $B$ be $A$ or a pattern forced by $A$ such that the period of $B$, denoted by $m$, is maximal in the sense of the Šarkovskiı̌ order in $\operatorname{Per}(A) \cup\{|A|\}$, i.e. $m \triangleright l$ for all $l \in \operatorname{Per}(A) \cup\{|A|\}$ with $l \neq m$. From the Sarkovskiı theorem it follows that $\operatorname{Per}(A) \cup\{|A|\}=\{m\} \cup\{l \in \mathbb{N}: m \triangleright l\}$. Such a maximal period must exist due to Lemma 4.9.

From Theorem 5.1, it follows that for $r$ sufficiently large, there exists $\lambda_{1}>0$ such that for all $|\lambda|<\lambda_{1}$ the map $F_{\lambda}$ has periodic points of all periods in $\{l \in \mathbb{N}: m \triangleright l\}=\operatorname{Per}(A) \cup\{|A|\} \backslash\{m\}$ in the set $I \times B_{n}(r)$, where $I$ is the closed interval containing the orbit realizing pattern $A$. If $m=|A|$, then we already have all periods from $\operatorname{Per}(A)$ for $F_{\lambda}$ with $|\lambda|$ sufficiently small. Therefore we can assume that $m \neq|A|$, and hence $B \neq A$ and $A \underset{\mathrm{pat}}{\Longrightarrow} B$. From the above we have all periods from $\operatorname{Per}(A) \backslash\{|B|\}$ for $F_{\lambda}$ with $|\lambda|$ sufficiently small and we have to prove that the pattern $B$ can be continued to a periodic orbit for $F_{\lambda}$ of period $|B|$.

Since $f$ exhibits a pattern $A, f$ exhibits the oriented pattern $\tilde{A}$ for one of the components of $A$. Since $A \underset{\text { pat }}{\Longrightarrow} B$, by Corollary 2.7.1 of [1], $\tilde{A} \underset{\mathrm{opat}}{\longrightarrow} \tilde{B}$ 
for one of the components of $B$. By Lemma 4.8 applied to $\tilde{A} \underset{\text { opat }}{\Longrightarrow} \tilde{B}$ and $f$, there exist closed proper intervals $J_{0}, J_{1}, \ldots, J_{m}$, where $m=|B|$, such that the following loop of 1-dim covering relations holds:

$$
J_{0} \stackrel{f}{\rightarrow} J_{1} \stackrel{f}{\rightarrow} \cdots \stackrel{f}{\rightarrow} J_{m-1} \stackrel{f}{\rightarrow} J_{m}
$$

such that

$$
\begin{aligned}
J_{0} \subset J_{m}, & \\
J_{i} \cap J_{j}=\emptyset & \text { for all } 0 \leq i, j \leq m-1 \text { with } i \neq j, \\
f^{m}(t) \neq t & \text { for all } t \in J_{0} \cap \partial\left(J_{m}\right) .
\end{aligned}
$$

Hence $f^{m}\left(J_{0}\right) \supset J_{m}$. Thus there exist $a, b \in J_{0}$ such that $f^{m}(a)=\min \left(J_{m}\right)$ and $f^{m}(b)=\max \left(J_{m}\right)$. Since there is no $t \in J_{0} \cap \partial\left(J_{m}\right)$ such that $f^{m}(t)=t$, it follows that $f^{m}(a)<a$ and $f^{m}(b)>b$.

We have two cases: $a<b$ and $a>b$. Assume first that $a<b$. Observe that $[a, b] \subset \operatorname{int}\left(J_{m}\right)$. By using Lemma 4.7, it is easy to find intervals $K_{i} \subset J_{i}$ for $i=0, \ldots, m-1$ such that

$$
K_{0} \stackrel{f}{\Rightarrow} K_{1} \stackrel{f}{\Rightarrow} \cdots \stackrel{f}{\Rightarrow} K_{m-1} \stackrel{f}{\Rightarrow} K_{0} .
$$

We set $N_{i}=K_{i} \times \overline{B_{n}(r)}$. By the continuity of $F_{\lambda}$ as a function of $\lambda$, there exists $0<\lambda_{2} \leq \lambda_{1}$ such that for all $|\lambda|<\lambda_{2}$,

$$
N_{0} \stackrel{F_{\lambda}}{\Longrightarrow} N_{1} \stackrel{F_{\lambda}}{\Longrightarrow} \cdots \stackrel{F_{\lambda}}{\Longrightarrow} N_{m-1} \stackrel{F_{\lambda}}{\Longrightarrow} N_{0} .
$$

From Theorem 4.3, we obtain a periodic point of period $m$ for $F_{\lambda}$ with $|\lambda|<\lambda_{2}$.

Consider now the case $a>b$. It is easy to see that we can apply Lemma 4.10 to obtain a periodic point of period $m$ for $F_{\lambda}$ with $|\lambda|$ sufficiently small.

Next, we give an example.

EXAmple 5.2. Consider the case when $f$ has a period- $(m+k)$ point $x_{0}$. Assume that the points of the orbit satisfy either

$$
x_{m+k-1}<\cdots<x_{k+1}<x_{k}<x_{0}<x_{1}<x_{2}<\cdots<x_{k-1}
$$

or the same with all inequalities reversed, where $x_{i}=f^{i}\left(x_{0}\right)$ for $i \geq 0$. By Theorems 2 and 3 of [6], the set of all periods of patterns which are forced by the above pattern is $\mathbb{N}$. By Theorem 2.2, for all sufficiently small $|\lambda|$, the high-dimensional map $F_{\lambda}$ has periodic points of all periods.

\section{Proof of Theorem 2.3 and an example}

Proof of Theorem 2.3. We will only prove the theorem when $f(\alpha) \leq$ $\alpha<\beta<f(\beta)<f^{2}(\beta)$ and $f^{3}(\beta) \leq \alpha$; the proof for the case with all 
inequalities reversed is similar. We set $I=\left[\alpha, f^{2}(\beta)\right], I_{0}=[\alpha, f(\beta)]$ and $I_{1}=\left[f(\beta), f^{2}(\beta)\right]$. We have

$$
I_{0} \stackrel{f,+1}{\longrightarrow} I_{j}, \quad I_{1} \stackrel{f,-1}{\longrightarrow} I_{j}, \quad j=0,1, \quad I \subset f(I) .
$$

We will now show that the map $F_{0}$ has a $(2, \infty)$-nested sequence of topological horseshoes such that $N_{0}^{s} \subset I_{0}^{s} \times \overline{B_{n}(r)}$ and $N_{1}^{s} \subset I_{1}^{s} \times \overline{B_{n}(r)}$, where $r$ is a positive number greater than the maximum of $\|g\|$ on $I$ for case (i) and on $I \times S$ for case (ii). Indeed, two preimages of $f(\beta)$ exist: one in $I_{0}$ (this could be $\beta$ ), denoted by $\gamma_{0}$, and another one in $I_{1}$, denoted by $\gamma_{1}$. We set $I_{0}^{2}=\left[\gamma_{0}, f(\beta)\right]$ and $I_{1}^{2}=\left[f(\beta), \gamma_{1}\right]$. Observe that $I_{i}^{2} \subset \operatorname{int}(I)$ and $I \subset f^{2}\left(I_{i}^{2}\right)$ for $i=0,1$. Using this and $I \subset f(I)$ we can easily construct two nested sequences of intervals $I_{i}^{s+1} \subset I_{i}^{s}$ for $s=2,3, \ldots$ and $i \in\{0,1\}$ such that $f^{s}\left(I_{i}^{s}\right)=I$. It is immediate to verify that we have a $(2, \infty)$-nested sequence of topological horseshoes for $F_{0}$, where $N_{i}^{s}=I_{i}^{s} \times \overline{B_{n}(r)}$.

For any $k>2$ there exists $\lambda_{k}>0$ such that for any $|\lambda|<\lambda_{k}$, the map $F_{\lambda}$ has a $(2, k)$-nested sequence of horseshoes. It is enough to take $k=3$ and apply Theorem 4.5 with $p=2$. Thus, there exists an integer $M(p)$ such that the map $F_{\lambda}$ with $|\lambda|<\lambda_{3}$ has periodic points of all periods greater than $M(p)$. There are a finite number of periods left and we need to continue them one by one using Lemma 4.10, which can be done by considering the following loops of covering relations for $f: I_{1} \stackrel{f,-1}{\longrightarrow} I_{1}$ for period one and $I_{1} \stackrel{f,-1}{\longrightarrow} I_{0} \stackrel{f,+1}{\longrightarrow} I_{0} \stackrel{f,+1}{\longrightarrow} I_{0} \stackrel{f,+1}{\longrightarrow} \cdots \stackrel{f,+1}{\longrightarrow} I_{0} \stackrel{f,+1}{\longrightarrow} I_{1}$ for other periods. From Lemma 4.10 we obtain, for $\lambda$ sufficiently small, a periodic orbit $z_{0}, z_{1}, \ldots, z_{p-1}, z_{0}=F_{\lambda}^{p}\left(z_{0}\right)$, where $p$ is the length of the loop, such that

$$
\begin{aligned}
z_{0} & \in \operatorname{int}\left(I_{1}\right) \times B_{n}(r), \\
z_{i}=F_{\lambda}^{i}\left(z_{0}\right) & \in \operatorname{int}\left(I_{0}\right) \times B_{n}(r) \quad \text { for all } 1 \leq i \leq p-1 .
\end{aligned}
$$

Since $\operatorname{int}\left(I_{0}\right) \cap \operatorname{int}\left(I_{1}\right)=\emptyset$ we see that the principal period of $z_{0}$ is equal to $p$.

The following example shows that if an interval map has a fixed point associated with a homoclinic-like orbit then any small multidimensional perturbation has periodic points of all periods.

EXAmple 6.1. Consider the case when $f$ has a fixed point $\alpha$ associated with an orbit $\left\{\beta_{i}\right\}_{i=-\infty}^{\infty}$ satisfying $f\left(\beta_{i}\right)=\beta_{i+1}$ for all integers $i$, and $\beta_{3} \leq$ $\alpha<\beta_{0}<\beta_{1}<\beta_{2}$. By Theorem 2.3, for all sufficiently small $|\lambda|$, the highdimensional map $F_{\lambda}$ has periodic points of all periods. Notice that if, in addition, $\lim _{i \rightarrow \pm \infty} \beta_{i}=\alpha$, then $\beta_{0}$ is a homoclinic point for $\alpha$ and it forces certain patterns for $f$; refer to $[2,5,6]$.

Acknowledgments. The first author was supported in part by NSC grant 96-2115-M-009-004-MY3. 
This work was done when the second author visited NCTS (Hsinchu, Taiwan). He was supported in part by Polish Ministry of Science and Higher Education grant N20102431/2163.

\section{References}

[1] L. Alsedà, J. Llibre and M. Misiurewicz, Combinatorial Dynamics and Entropy in Dimension One, 2nd ed., Adv. Ser. Nonlinear Dynam. 5, World Sci., Singapore, 2000.

[2] N. P. Bhatia and W. O. Egerland, A refinement of Šarkovskiù's theorem, Proc. Amer. Math. Soc. 102 (1988), 965-972.

[3] L. S. Block and W. A. Coppel, Dynamics in One Dimension, Lecture Notes in Math. 1513, Springer, Berlin, 1992.

[4] A. Blokh, Rotation numbers, twists and a Sharkovskiu-Misiurewicz-type ordering for patterns on the interval, Ergodic Theory Dynam. Systems 15 (1995), 1-14.

[5] L. A. V. Carvalho, On an extension of Sarkovskiǔ's order, J. Math. Anal. Appl. 138 (1989), 52-58.

[6] B.-S. Du and M.-C. Li, A refinement of Sharkovskii's theorem on orbit types characterized by two parameters, J. Math. Anal. Appl. 278 (2003), 77-82.

[7] A. N. Šarkovskiı̌, Coexistence of cycles of a continuous map of a line into itself, Ukrain. Mat. Ž. 16 (1964), 61-71 (in Russian).

[8] - Coexistence of cycles of a continuous map of a line into itself, Int. J. Bifur. Chaos 5 (1995), 1263-1274.

[9] P. Stefan, A theorem of Śarkovskii on the existence of periodic orbits of continuous endomorphisms of the real line, Comm. Math. Phys. 54 (1977), 237-248.

[10] P. Zgliczyński, Sharkovskii's Theorem for multidimensional perturbations of onedimensional maps, Ergodic Theory Dynam. Systems 19 (1999), 1655-1684.

[11] - Sharkovskiı Theorem for multidimensional perturbations of one-dimensional maps II, Topol. Methods Nonlinear Anal. 14 (1999), 169-182.

[12] —, Multidimensional perturbations of one-dimensional maps and stability of Šarkovskiu ordering, Int. J. Bifur. Chaos 9 (1999), 1867-1876.

Ming-Chia Li

Department of Applied Mathematics

National Chiao Tung University

1001 Ta Hsueh Road

Hsinchu 300, Taiwan

E-mail: mcli@math.nctu.edu.tw
Piotr Zgliczyński

Institute of Computer Science Jagiellonian University Łojasiewicza 6 30-348 Kraków, Poland E-mail: umzglicz@cyf-kr.edu.pl zgliczyn@ii.uj.edu.pl

Received 9 February 2009;

in revised form 1 August 2009 